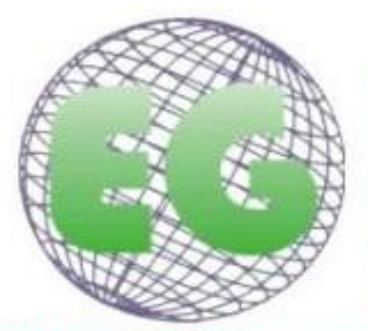

ISSN 1695-6141 $N^{\circ} 47$

www.um.es/eglobal/

\title{
ORIGINALES
}

\section{Estudio de la satisfacción laboral en los equipos de atención primaria en un área sanitaria de Asturias}

Study of job satisfaction in primary healthcare in an area of Asturias

\author{
Andrea Rodríguez-Alonso ${ }^{1}$ \\ Patricia Gómez-Fernández ${ }^{2}$ \\ Ricardo de-Dios del-Valle ${ }^{3}$ \\ ${ }^{1}$ Enfermera Especialista en Enfermería Familiar y Comunitaria, Centro de Salud "El Coto", Área V, \\ SESPA \\ ${ }^{2}$ Enfermera Especialista en Enfermería Familiar y Comunitaria, Centro de Salud "Contrueces", Área V, \\ SESPA \\ ${ }^{3}$ Médico de Admisión y Documentación Clínica, Hospital Universitario Central de Asturias, SESPA \\ E-mail: andrearodriguezalonso@gmail.com
}

http://dx.doi.org/10.6018/eglobal.16.3.256641

Recibido: $15 / 04 / 2016$

Aceptado: 19/07/2016

\section{RESUMEN:}

Objetivo: Valorar nivel de satisfacción laboral de los profesionales que trabajan en Atención Primaria en un Área de Asturias y los factores asociados.

Metodología: Estudio descriptivo transversal. Población de estudio: profesionales de cualquier categoría que trabajen en Centros de Salud del Área V (Asturias). Muestra: toda la población. Utilizamos el cuestionario validado Font-Roja AP y recogemos variables sociodemográficas y laborales. Las variables cuantitativas se describen con media y desviación estándar, las cualitativas con distribución porcentual de frecuencias. Se buscan posibles relaciones entre satisfacción laboral y resto de variables usando Chi-Cuadrado, t-Student y ANOVA.

Resultados: Tasa de respuesta: $57.22 \%$ (329 de 575). $72.3 \%$ mujeres. Edad media: 50,8 años (DE: 10.09). El 83.9\% se autodeclaran como satisfechos (IC95\%:83.5-91.2). Sólo el 62.6\% (IC 95\%: 57.468.2) están satisfechos calculando la Satisfacción Media Global (SMG) derivada de Font-Roja. Dimensiones del Font-Roja con mayor satisfacción "relación con los compañeros" y "competencia profesional". Menor satisfacción: "promoción profesional" y "tensión relacionada con trabajo". Todas las categorías otorgan baja puntuación al sueldo, siendo las peores administrativos y celadores $(p<0,001)$. En el análisis bivariante entre SMG y variables sociodemográficas, hay asociación estadísticamente significativa con la docencia, $68.8 \%$ satisfechos en centros docentes ( $p 0,039$ ) y con categoría profesional, siendo los más satisfechos los MIR/EIR $88,9 \%$ y los menos los médicos $46,1 \%$ (p: 0.009 ).

Conclusiones: La mayoría de los profesionales se encuentran satisfechos laboralmente. Presentan una mayor satisfacción autodeclarada que la que se deduce de la medición SMG en Font-Roja. Áreas de mejora: sueldo, promoción profesional y tensión en el trabajo.

Palabras clave: Satisfacción en el trabajo; Atención Primaria de Salud; Personal de Salud. 


\section{ABSTRACT:}

Objective: Evaluate the level of job satisfaction of professionals working in Primary healthcare in Asturias and associated factors.

Methodology: Cross-sectional descriptive study.Study Population: professionals of any category working at Health Centers in the province of V (Asturias). Sample: Whole population. We used the validated Red Font AP "Roja Font" questionnaire and gathered it up with variables socio-demographic and work variables. The quantitative variables are described with average and standard deviation, the qualitative with frequency distribution percentage. Possible relationships are sought between job satisfaction and the remaining variables using chi-square, t-Student and ANOVA.

Results: Response rate: $57.22 \%$ (329 out of 575). $72.3 \%$ women. Average age: 50.8 years (From: 10.09). $83.9 \%$ self-reported as satisfied (95\% Cl: $83.5-91.2)$. Only $62.6 \%$ (95\% Cl: $57.4-68.2)$ were satisfied by calculating the Global Average Satisfaction (GAS) derived from "Font-Roja". Dimensions of the "Font-Roja" with greater satisfaction: "relationship with coworkers" and "professional competence". Lower satisfaction: "professional promotion" and "work-related tension". All the categories gave low score to the salary, being the worse administrative and hospital attendant $(p<0,001)$. In the bivariate analysis between GAS and socio-demographic variables, there was a statistically significant association with teaching, 68.8\% satisfied in the educational centers (p .039) and with professional category, remaining the most satisfied being MIR / EIR $88.9 \%$ and the least were doctors with $46.1 \%$ (w: 0.009 ).

Conclusions: The majority of professionals are satisfied occupationally. They present a higher selfreported satisfaction than that derived from the GAS measurement in Font-Roja.Areas of improvement: wages, professional promotion and development and tension at work.

Keywords: Job satisfaction; Primary health care; Health personnel.

\section{INTRODUCCIÓN}

El trabajo juega un papel destacado en la vida del hombre. La mayoría de las personas pasan una tercera parte del día trabajando y lo hacen durante la mitad de su vida. Sin embargo, no fue hasta el pasado siglo XX cuando se comenzó a estudiar el trabajo desde el punto de vista científico, relacionando este tema con diferentes aspectos. Frank Taylor, en 1911, asumía que la satisfacción en el trabajo estaba totalmente relacionada con el salario recibido, es decir, con las recompensas. Más tarde, en los años treinta, aparecen estudios industriales que comienzan a dar importancia al bienestar del trabajador. Happock consideró que la satisfacción en el trabajo formaba parte de la satisfacción general con la vida y estaba relacionada con la habilidad del individuo para adaptarse a situaciones y comunicarse con otros, con el nivel socioeconómico y con la preparación de la persona para ese tipo de trabajo. ${ }^{1}$

En 1945, Elton Mayo aseguró que la interacción del individuo con el grupo era el determinante más importante de la satisfacción en el trabajo y la situó por encima de otros factores que también influían en la satisfacción, como la seguridad, estima, afiliación, interés intrínseco por el trabajo, logros, etc. Este estudio fue el primero que se realizó desde una perspectiva psicológica. ${ }^{2}$

Las teorías de la motivación han supuesto otra influencia importante en los estudios de satisfacción en el trabajo. El psicólogo humanista Maslow desarrolló en 1954 una jerarquía de necesidades humanas, situando en el lugar más básico las necesidades que son vitales para sobrevivir y en el lugar más elevado aquellas que permiten el desarrollo intelectual de la persona. Basándose en esta teoría, la satisfacción ha sido enfocada por los investigadores sobre todo desde la perspectiva de una necesidad de realización personal. $^{3}$

Herzberg desarrolló una teoría de la satisfacción en el trabajo basada en la jerarquía de Maslow, en la que concluía que no todos los factores influyen en la satisfacción; los correspondientes a las necesidades más básicas cuentan si no están cubiertos, 
mientras que otros factores más elevados incrementan la satisfacción al conseguirlos. Esta teoría de higiene y motivación ha prevalecido en los estudios de satisfacción de la naturaleza del trabajo y ha conformado las bases del desarrollo de la valoración de dicha satisfacción ${ }^{4}$.

En 1976, Locke definió la satisfacción laboral como "un estado emocional positivo o placentero de la percepción subjetiva de las experiencias laborales del trabajador". Se trataría de una orientación afectiva que un empleado tiene hacia su trabajo, que podría ser considerada como un sentimiento global acerca del trabajo o una agrupación de actitudes relacionadas con varios aspectos del trabajo. En definitiva, se podría decir entonces que una persona está satisfecha con su trabajo cuando está a gusto en él y tiene una actitud positiva individual hacia el trabajo y las condiciones en que se realiza ${ }^{5}$.

La satisfacción profesional constituye el primer indicador de calidad de vida laboral y, como ya hemos visto, existen una multiplicidad de variables que pueden incidir en ella ${ }^{6}$.

Con respecto al ámbito sanitario, analizando el devenir de los cuidados de salud en nuestra sociedad observamos una constante evolución de los conocimientos científicos, los medios técnicos, el propio sistema sanitario, una modificación de los patrones epidemiológicos, así como una evolución de la pirámide de población y las necesidades que demandan los ciudadanos del Sistema Nacional de Salud. Todo ello, converge en la aparición de un nuevo y complejo modelo de cuidados, que ha de dar una respuesta eficaz y segura a las necesidades de nuestros ciudadanos ${ }^{6}$. En las organizaciones sanitarias el factor humano juega un papel fundamental para la concesión con "calidad" de estos servicios, ya que a diferencia de otras organizaciones, éstos se encuentran estrechamente relacionados con el trato personal. Varios estudios mostraron que para los usuarios, la calidad está representada y definida por las características del proceso de atención, en donde el factor principal del proceso es el trato personal que se les brinda ${ }^{7,8}$. La satisfacción del cliente o usuario exige el compromiso del personal y se considera que "la satisfacción del empleado influye sobre la del cliente" (a mayor satisfacción laboral, mayor percepción de la calidad por parte del usuario), reconociendo que una atmósfera interna de calidad crea un ambiente que lleva a satisfacer a los clientes externos o usuarios en términos de alta eficacia de la intervención, uso adecuado de la técnica y la tecnología y relación interpersonal con calidez humana ${ }^{9}$.

Los profesionales sanitarios han sido, por tanto, un grupo bastante estudiado en cuestiones de satisfacción. Se consideran como profesiones particularmente estresantes ya que poseen altos niveles de responsabilidad, relaciones interpersonales y exigencias sociales, lo que afecta tanto la salud y el bienestar personal como a la satisfacción laboral y colectiva ${ }^{10}$.

Dado que la satisfacción en el trabajo de los profesionales de la salud es uno de los indicadores que condicionan la calidad asistencial, la medición rutinaria de la satisfacción laboral y el diseño de acciones de mejora para corregir aquellos aspectos con peores resultados deben ser una práctica obligada para cualquier equipo de gestión $^{6,11,12}$. La satisfacción laboral de los profesionales se puede igualar en importancia a la preparación científico-técnica que posean o a tener en su disposición una determinada tecnología ${ }^{13}$. La implantación de un modelo de calidad total requiere por tanto, conocer la satisfacción de los trabajadores ${ }^{14}$. 
Varios estudios de investigación revelan que la satisfacción de los profesionales sanitarios está estrechamente relacionada con el nivel de autonomía, las condiciones laborales, el respeto y reconocimiento al trabajo que se realiza, una plantilla adecuada y suficiente, las buenas relaciones entre los miembros del equipo, el sueldo, el compromiso con la organización y la implicación profesional. Aspectos todos estrechamente relacionados con el estilo y modelo de gestión establecido por los directivos de la organización ${ }^{15}$.

En nuestro país se han realizado varias investigaciones al respecto en el entorno de los trabajadores de los Servicios de Salud. En el ámbito de Atención Primaria podemos encontrar dos trabajos realizados en la Comunidad de Madrid ${ }^{16,17}$ en los que analizaban al personal de todas las categorías que trabajan en los Centros de Salud: Médicos, Enfermería, otras profesiones sanitarias (Fisioterapeutas, Matronas, etc.) y personal administrativo. En 2013 se llevó a cabo una investigación en Asturias que estudiaba profesionales de varias categorías profesionales procedentes tanto de Atención Primaria como Especializada que estuvieran desarrollando su labor en Unidades de Gestión Clínica ${ }^{18}$.

Otros trabajos estudiaron la satisfacción de sólo una categoría profesional en concreto, como pueden ser trabajos realizados en Asturias ${ }^{19}$, Málaga ${ }^{20}$ o Barcelona ${ }^{21}$, que se circunscribían al personal de Medicina, $u$ otros realizados por unas compañeras en Galicia ${ }^{22}$ y en Soria $^{23}$ que se dedicaban al personal de Enfermería en exclusiva.

Nuestra intención en el presente trabajo fue valorar y analizar a todo el conjunto de trabajadores de los Centros de Salud, por lo que incluimos personal de Medicina y Enfermería, otras profesiones de ámbito sanitario y personal administrativo. El Servicio de Salud del Principado de Asturias (SESPA) está dividido geográficamente en ocho áreas sanitarias y nuestro trabajo se llevó a cabo en el Área Sanitaria V (Gijón).

El objetivo general de nuestro trabajo fue valorar el nivel de satisfacción laboral de los distintos profesionales que trabajan en Atención Primaria en el Área V. Como objetivos específicos buscamos conocer las características sociodemográficas de los trabajadores de Atención Primaria del Área $\mathrm{V}$ y analizar los aspectos que tienen mayor influencia sobre la satisfacción laboral.

\section{MATERIAL Y MÉTODO}

Diseño: Estudio descriptivo transversal.

Ámbito: Atención Primaria del Área V (Gijón) del Servicio de Salud del Principado de Asturias.

Población de estudio: Profesionales de todas las categorías (sanitarias y no sanitarias) que desarrollan su trabajo en los centros de salud del Área V. Criterios de exclusión:

a) Personal que lleve trabajando menos de 6 meses en Atención Primaria en el momento de la recogida de los datos.

b) Personal que se encuentre en situación de incapacidad laboral, vacaciones, etc. durante el período de la recogida de datos. 
No se realiza muestra, se pasa el cuestionario a todos los profesionales.

Período de estudio: 15 de septiembre a 31 de noviembre de 2014.

Fuentes de Información: Cuestionario de elaboración propia para la recogida de variables sociodemográficas y cuestionario de Font Roja AP para evaluar la satisfacción laboral. El cuestionario es anónimo y autoadministrado en privado. La entrega y recogida se realizó a través de los Coordinadores de los Centros. Los cuestionarios eran depositados en urna opaca situada en la Unidad Administrativa del Centro de Salud. La participación era voluntaria. Previo a la consulta se solicitó por escrito autorización a la Gerencia y Dirección de enfermería del Área Sanitaria, aportando copia de nuestro cuestionario.

Variables recogidas en cuestionario de elaboración propia: Variables sociodemográficas: sexo, edad, estado civil, no de hijos. Variables laborales: centro de salud, tipo de contrato, tiempo trabajado en atención primaria y en el centro de salud, promedio de pacientes atendidos al día. Variables sobre satisfacción laboral: "Satisfacción autodeclarada" mediante la introducción de una pregunta directa formulada de la siguiente manera: “ ¿En general, se encuentra satisfecho/a del trabajo que desempeña?”, con opciones válidas si/no.

Cuestionario Font-Roja: Este cuestionario validado, que determina la satisfacción en los profesionales en centros hospitalarios, fue adaptado a las condiciones de trabajo de Atención Primaria, pasando a denominarse cuestionario Font Roja AP por J. Aranaz ${ }^{24}$. Consta de 24 ítems y explora 9 dimensiones que determinan la satisfacción de los profesionales.

Cada uno de los ítems es valorado mediante una escala Likert, cuyos valores oscilan entre 1 (totalmente en desacuerdo) y 5 (totalmente de acuerdo). Existen ítems que a mayor puntuación indican mayor satisfacción $(7,8,9,10,11,12,13,14,15,16,17$ y 19), y otros que a mayor puntuación nos indica una menor satisfacción $(1,2,3,4,5,6$, $18,20,21,22,23$ y 24$)$.

El resultado general de este cuestionario se expresa en un índice que se denomina Satisfacción Media Global (SMG), el cual resulta de calcular la diferencia entre los ítems del Cuestionario Font Roja que forman parte de las dimensiones que aumentan la satisfacción menos los que pertenecen a las que la disminuyen, dividiendo después el resultado entre los 24 ítems totales. De este cálculo deriva un resultado que oscila entre -2 y 2 puntos, permitiendo diferenciar las personas en satisfechos $(>0)$, indiferentes o neutros $(=0)$ o no satisfechos $(<0)$. EI SMG se constituye como la variable dependiente del trabajo.

Análisis estadístico: Se describe la muestra utilizando para las variables cuantitativas la media y la desviación típica, y para las variables cualitativas la distribución porcentual de frecuencias. Se realizó un primer análisis para comprobar el grado de concordancia entre las diversas formas de medir la satisfacción (Satisfacción Media Global, Ítem número 7 del cuestionario y satisfacción autodeclarada por el profesional) mediante el Índice Kappa. Se llevó a cabo un análisis univariante buscando la influencia de las variables independientes en la satisfacción laboral (resultado del Cuestionario de Font Roja AP (SMG) con la prueba de la Chi-cuadrado para las variables cualitativas y el test de Student, ANOVA u otras pruebas no paramétricas para las variables cuantitativas. Para comprobar qué variables se asocian de forma 
independiente con la variable satisfacción laboral (SMG) se realizó un análisis de regresión logística binaria (método de introducir); las variables seleccionadas para el modelo fueron aquellas que en el análisis univariante alcanzaron una significación estadística de $p<0.2$. Se define como nivel de significación estadística el $5 \%$. Se exponen los intervalos de confianza del $95 \%$ de los resultados más relevantes. El análisis se realizó con el paquete estadístico SPSS v.15.0.

\section{RESULTADOS}

\section{Análisis descriptivo del conjunto de participantes.}

Se distribuyó la encuesta entre todos los profesionales de los equipos de Atención Primaria, un total de 575 personas en el período de estudio. La tasa de respuesta fue del $57.22 \%$.

- Variables sociodemográficas.

Mayoritariamente son mujeres (72.3\%), edad media de 50.8 años y el $64.4 \%$ estaban casados o tenían pareja estable. La mayor parte tienen hijos (65.3\%).

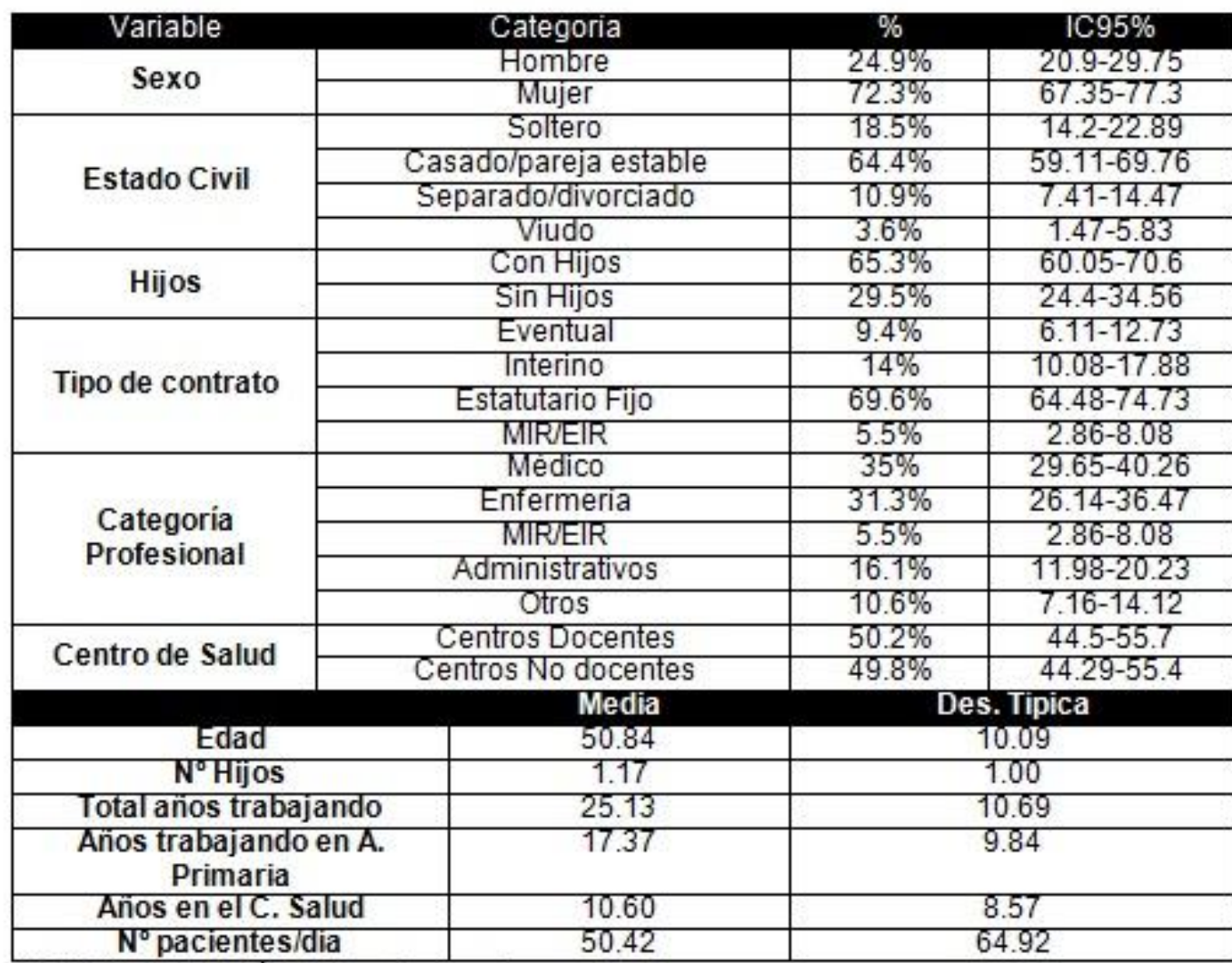

Tabla I. Descripción de nuestra muestra.

- Variables laborales.

La mayoría era personal estatutario fijo (69.6\%). Llevan una media de 25 años trabajando, de los cuales 17.3 años han ejercido en Atención Primaria. La presión asistencial media (pacientes atendidos al día) es de 50.42. Existe una gran variabilidad en el número de pacientes/día entre las diferentes categorías profesionales, así la presión asistencial de los médicos es de 37.53, frente a 21.85 de las enfermeras, 29.83 de MIR/EIR y 205.45 de personal administrativo. La variabilidad existe también dentro de cada categoría, así vemos que los pacientes atendidos al día por un médico oscilan entre un 
mínimo de 10 a un máximo de 70, y los atendidos por enfermería oscilan entre un mínimo de 12 y un máximo de 40, o en el caso de los MIR/EIR de 15 a 40.

\section{Análisis de la Satisfacción Laboral: Concordancia.}

En el presente estudio se ha valorado la satisfacción laboral mediante tres instrumentos: el primero y fundamental, la puntuación global del Cuestionario Font Roja AP (SMG) que clasifica a los profesionales en tres categorías (satisfecho, insatisfecho, indiferente) y que es el resultado final de la puntuación obtenida en los 24 ítems del mismo; el segundo es el resultado de la respuesta al ítem número 7 del citado cuestionario ("En mi trabajo me encuentro satisfecho"); y el tercero y último, con una pregunta cerrada que se introdujo en el cuestionario de recogida que pretende reflejar la "satisfacción autodeclarada" y que pregunta directamente a los profesionales si en general se encuentran satisfechos con su trabajo o no.

En primer lugar valoramos la concordancia existente entre los tres instrumentos, para lo que calculamos el índice Kappa. Observamos que existe una discordancia entre la satisfacción autodeclarada y el resultado del ítem número 7, así como entre el ítem número 7 y el resultado global del cuestionario (SMG). Existe concordancia entre satisfacción autodeclarada y la puntuación global del cuestionario (SMG) aunque el grado de acuerdo es bajo (Kappa entre 0.2 y 0.4 )

\begin{tabular}{|c|c|c|c|c|}
\hline & $\begin{array}{c}\text { Valor índice } \\
\text { Kappa }\end{array}$ & $\begin{array}{c}\text { Error típ. } \\
\text { asint. }\end{array}$ & $\begin{array}{c}\text { T } \\
\text { aproximada }\end{array}$ & $\begin{array}{c}\text { Sig. } \\
\text { Aproximada }\end{array}$ \\
\hline $\begin{array}{c}\text { Satisfacción Media Global } \\
\text { (SMG) } \\
\text { Con }\end{array}$ & 0.310 & 0.052 & 6.62 & 0.000 \\
Satisfacción autodeclarada & -0.287 & 0.049 & -8.036 & 0.000 \\
\hline Item 7 con SMG & -0.221 & 0.045 & -10.865 & 0.000 \\
\hline Item 7 con S. Autodeclarada & & &
\end{tabular}

Tabla ll. Acuerdo entre herramientas de medida. Indiœ Kappa.

\section{Análisis de la Satisfacción Laboral: Resultados.}

En relación con la "satisfacción autodeclarada" observamos que el $83.9 \%$ de los participantes respondieron que se encontraban satisfechos con el trabajo que desarrollaban frente al $12.2 \%$ que no se sentían satisfechos.

Según la puntuación del ítem 7 del Cuestionario, un 66.5\% estaban satisfechos, $19.6 \%$ indiferentes y $14 \%$ no satisfechos.

Y, por último, siguiendo el SMG el $62.4 \%$ de nuestros profesionales estudiados se encuentran satisfechos, un $2.1 \%$ indiferentes, y un $35.5 \%$ insatisfechos.

\begin{tabular}{|c|c|c|c|}
\hline \multicolumn{3}{|c|}{ Comparación de los Resultados de Salud Laboral según la herramienta de medida. } \\
\hline & Categoría & $\%$ & IC95\% \\
\hline \multirow{3}{*}{ SMG (24 items) } & Satisfecho (>0) & 62.4 & $56.98-67.79$ \\
\cline { 2 - 4 } & Indiferente (0) & 2.1 & $0.42-3.86$ \\
\cline { 2 - 4 } & No Satisfecho (<0) & 35.5 & $30.14-40.81$ \\
\hline \multirow{2}{*}{$\begin{array}{c}\text { Item 7 "En mi trabajo } \\
\text { me encuentro } \\
\text { satisfecho" }\end{array}$} & Satisfecho (4-5) & $66.5 \%$ & $61.15-71.8$ \\
\cline { 2 - 4 } & Indiferente (3) & $19.6 \%$ & $15.08-24.05$ \\
\cline { 2 - 4 } & No satisfecho (1-2) & $14 \%$ & $10.03-17.92$ \\
\hline \multirow{2}{*}{$\begin{array}{c}\text { Satisfacción } \\
\text { autodeclarada }\end{array}$} & Satisfecho & $83.9 \%$ & $79.77-88.01$ \\
\cline { 2 - 4 } & No Satisfecho & $12.2 \%$ & $8.48-15.84$ \\
\hline
\end{tabular}

Tabla III. Resultados de Satisfacción Laboral 


\begin{tabular}{|c|c|c|c|}
\hline Dimensión & item & Media & $\begin{array}{l}\text { Desv. } \\
\text { Típica }\end{array}$ \\
\hline \multirow{4}{*}{ Satisfacción por el trabajo } & F.7. "En mi trabajo me encuentro satisfecho" & 3.71 & 1.12 \\
\hline & F.10. "Tengo interés por las cosas que realizo" & 4.38 & 0.81 \\
\hline & $\begin{array}{l}\text { F.11. "Tengo la sensación de que lo que hago } \\
\text { vale la pena" }\end{array}$ & 3.88 & 0.99 \\
\hline & $\begin{array}{l}\text { F.16. "Ocupo el puesto que merezco por } \\
\text { capacidady preparación" }\end{array}$ & 3.55 & 1.19 \\
\hline \multirow{5}{*}{$\begin{array}{l}\text { Tensión relacionada con } \\
\text { el trabajo }\end{array}$} & $\begin{array}{l}\text { F.2. "Tengo responsabilidad a la hora de tomar } \\
\text { decisiones" }\end{array}$ & 4.07 & 1.13 \\
\hline & $\begin{array}{l}\text { F.3. Al final de la jornada me encuentro muy } \\
\text { cansado }\end{array}$ & 3.52 & 1.11 \\
\hline & $\begin{array}{l}\text { F.4. "No desconecto del trabajo cuandollego a } \\
\text { casa" }\end{array}$ & 2.35 & 1.15 \\
\hline & $\begin{array}{l}\text { F.5. "En el trabajo diario me tengo que emplear } \\
\text { a fondo" }\end{array}$ & 4.19 & 0.88 \\
\hline & F.6. "Mi trabajo me altera el estado de ánimo" & 2.94 & 1.24 \\
\hline \multirow{3}{*}{ Competencia profesional } & $\begin{array}{l}\text { F.22. "Con frecuencia siento no estar capacitado } \\
\text { para mi trabajo" }\end{array}$ & 1.97 & 1.10 \\
\hline & $\begin{array}{l}\text { F.23. "Siento que no tengo recursos suficientes } \\
\text { para realizar mi trabajo tan bien como desearia" }\end{array}$ & 3.15 & 1.17 \\
\hline & $\begin{array}{l}\text { F.24. "La competitividad me causa estrés o } \\
\text { tensión". }\end{array}$ & 2.31 & 1.26 \\
\hline \multirow[b]{2}{*}{ Presión del trabajo } & F.18. "Me falta tiempo para realizar mi trabajo" & 3.17 & 1.24 \\
\hline & $\begin{array}{l}\text { F.20. "Creo que mi carga de trabajo es } \\
\text { excesiva" }\end{array}$ & 3.35 & 1.19 \\
\hline \multirow{3}{*}{ Promoción profesional } & $\begin{array}{l}\text { F.9. "Tengo oportunidad de aprender cosas } \\
\text { nuevas" }\end{array}$ & 3.52 & 1.13 \\
\hline & F.12. "Obtengo reconocimiento pormi trabajo" & 2.80 & 1.16 \\
\hline & $\begin{array}{l}\text { F.17. }{ }^{a} \text { Tengo muchas posibilidades de } \\
\text { promoción }\end{array}$ & 1.89 & 1.02 \\
\hline \multirow{2}{*}{$\begin{array}{l}\text { Relación interpersonal } \\
\text { con los jefes }\end{array}$} & F.13. "La relación con mis superiores es cordial" & 3.59 & 1.19 \\
\hline & F.19. "Sé lo que se espera de mí en el trabajo" & 3.60 & 1.05 \\
\hline $\begin{array}{l}\text { Relación interpersonal } \\
\text { con los compañeros }\end{array}$ & $\begin{array}{l}\text { F.14. "Las relaciones con los compañeros son } \\
\text { cordiales" }\end{array}$ & 4.18 & 0.85 \\
\hline \multirow{2}{*}{$\begin{array}{l}\text { Características } \\
\text { extrínsecas del estatus }\end{array}$} & $\begin{array}{l}\text { F.8. "Tengo independencia para organizar mi } \\
\text { trabajo" }\end{array}$ & 3.48 & 1.18 \\
\hline & F.15. "El sueldo es adecuado" & 2.53 & 1.11 \\
\hline \multirow[b]{2}{*}{ Monotonía Laboral } & F.1. "Mi trabajo no varía, resulta monótono" & 2.56 & 1.09 \\
\hline & $\begin{array}{l}\text { F. 21. "Los problemas personales de mis } \\
\text { compañeros me afectan" }\end{array}$ & 3.16 & 1.17 \\
\hline
\end{tabular}

Tabla IV. Resultados en los 24 ítems que componen el cuestionario de Font Roja AP. Se

señala el orden del ítem en el Cuestionario de Font Roia AP.

Si procedemos a analizar los resultados obtenidos en las nueve dimensiones de las que consta el cuestionario Font Roja AP, nos encontramos que las dimensiones con mayor porcentaje de profesionales satisfechos son la dimensión 1 "Satisfacción por el trabajo" y la dimensión 7 "Relación interpersonal con los compañeros", en las que un $84.8 \%$ y un $81.8 \%$ de los profesionales se encuentran satisfechos respectivamente.

Las dimensiones con mayor porcentaje de profesionales insatisfechos son: la dimensión 2 "Tensión relacionada con el trabajo", la dimensión 5 "Promoción profesional" y la dimensión 4 "Presión en el trabajo", donde el $66.3 \%$, el $52.9 \%$ y el $50.5 \%$ de los profesionales se encuentran insatisfechos respectivamente.

\section{Análisis de la relación entre SMG y el resto de variables.}

Analizamos la relación existente entre el SMG agrupado en categorías (Satisfecho, indiferente e insatisfecho) con las variables independientes. 


\begin{tabular}{|c|c|c|c|c|c|}
\hline Variable & Categoria & Satisfechos & $\begin{array}{c}\text { SMG } \\
\text { Indiferente }\end{array}$ & Insatisfecho & p \\
\hline \multirow{2}{*}{ Sexo } & Hombre & $60.5 \%$ & $1.2 \%$ & $38.3 \%$ & \multirow{2}{*}{ n.s. } \\
\hline & Mujer & $63.3 \%$ & $2.5 \%$ & $34.2 \%$ & \\
\hline \multirow{2}{*}{ Hijos } & Con hijos & $61.7 \%$ & $1.9 \%$ & $36.4 \%$ & \multirow{2}{*}{ n.s. } \\
\hline & Sin hijos & $66 \%$ & $4.1 \%$ & $29.9 \%$ & \\
\hline \multirow{4}{*}{ Estado Civil } & Soltero & $67.2 \%$ & $1.6 \%$ & $31.1 \%$ & \multirow{4}{*}{ n.s. } \\
\hline & Casado & $61.6 \%$ & $2.4 \%$ & $36 \%$ & \\
\hline & Separado & $58.3 \%$ & $2.8 \%$ & $38.9 \%$ & \\
\hline & Viudo & $58.3 \%$ & $8.3 \%$ & $33.3 \%$ & \\
\hline \multirow{5}{*}{$\begin{array}{l}\text { Categoría } \\
\text { Profesional }\end{array}$} & Médico & $46.1 \%$ & $3.5 \%$ & $50.4 \%$ & \multirow{5}{*}{$<0.001$} \\
\hline & Enfermera & $70.9 \%$ & $3.9 \%$ & $25.2 \%$ & \\
\hline & MIRVEIR & $88.9 \%$ & $0 \%$ & $11.1 \%$ & \\
\hline & Administrativo & $57.7 \%$ & $0 \%$ & $42.3 \%$ & \\
\hline & Otros & $82.9 \%$ & $0 \%$ & $17.1 \%$ & \\
\hline \multirow{3}{*}{$\begin{array}{l}\text { Tipo de } \\
\text { contrato }\end{array}$} & Propietario/Interino & $61.3 \%$ & $2.9 \%$ & $35.8 \%$ & \multirow{3}{*}{0.047} \\
\hline & Eventual & $54.8 \%$ & $0 \%$ & $45.2 \%$ & \\
\hline & MIR/EIR & $88.9 \%$ & $0 \%$ & $11.1 \%$ & \\
\hline \multirow{2}{*}{ C. Salud } & Docente & $65.9 \%$ & $4.3 \%$ & $29.9 \%$ & \multirow{2}{*}{0.018} \\
\hline & No docente & $58.5 \%$ & $0.6 \%$ & $40.9 \%$ & \\
\hline \multicolumn{2}{|r|}{ Edad $^{3}$} & 50.04 & 54.12 & 52.03 & 0.161 \\
\hline \multicolumn{2}{|c|}{$N^{\circ} h_{i j o s}{ }^{2}$} & 1.15 & 1.5 & 1.19 & n.s. \\
\hline \multicolumn{2}{|c|}{ Años trabajados totales" } & 24.72 & 27.63 & 25.69 & n.s. \\
\hline \multicolumn{2}{|c|}{ Años trabajados en $\mathrm{AP}^{\mathrm{a}}$} & 15.88 & 19.38 & 19.82 & 0.003 \\
\hline \multirow{2}{*}{\multicolumn{2}{|c|}{$\begin{array}{l}\text { Años en C. Salud } \\
N^{\circ} \text { pacientes/dia }\end{array}$}} & 10.28 & 13.38 & 10.88 & n.S. \\
\hline & & 49.4 & 30.75 & Tabla V. Relación entre la Satisfacción Media Global y el resto de variables. "En las variables & 0.009 \\
\hline
\end{tabular}

Encontramos diferencias estadísticamente significativas entre la satisfacción global y los años que llevan trabajando en atención primaria, el número de pacientes atendidos al día, el tipo de contrato, la categoría profesional y el centro de salud (docente/no docente).

Respecto al número de años trabajando en Atención Primaria nos encontramos que los profesionales insatisfechos llevan más años trabajando en atención primaria (19.82) que los satisfechos (15.88). En relación con el tipo de contrato podemos observar como el porcentaje de insatisfechos en profesionales interinos o fijos es del $35.8 \%$ frente al $45.2 \%$ de los eventuales.

El porcentaje de insatisfechos en los profesionales que trabajan en centros de salud sin docencia es del $40.9 \%$ frente al $29.9 \%$ de los que trabajan en centros docentes.

Si tenemos en cuenta la presión asistencial observamos que en la presión asistencial media en profesionales insatisfechos es de 70.03 frente a 49.4 de los profesionales satisfechos.

Por último, las categorías profesionales con mayor porcentaje de insatisfechos son los médicos (50.4\%) y los administrativos (42.3\%) frente al $25.2 \%$ de las enfermeras o al $11.1 \%$ de los MIR/EIR. En el resto de las variables no observamos diferencias estadísticamente significativas.

Para evaluar el efecto de la edad, años trabajados en atención primaria, categoría profesional, centro de salud docente o no, tipo de contrato y número de pacientes atendidos sobre la satisfacción laboral (SMG) realizamos una regresión logística binaria. El modelo de regresión fue estadísticamente significativo $\left(X^{2}=42.834\right.$, $p<0.001)$. El modelo explica el $19.9 \%$ de la varianza en la satisfacción laboral $\left(R^{2}\right.$ de Nagelkerke $=0.199)$ y clasifica correctamente al $68.9 \%$ de los casos. La sensibilidad 
fue del $83.23 \%$, la especificidad del $45.63 \%$, el valor predictivo positivo del $71.28 \%$ y el valor predictivo negativo del $62.66 \%$. De las seis variables independientes, solamente dos lo fueron estadísticamente significativas, la categoría profesional $(\mathrm{OR}=3.833$; IC95\%=1.334-11.012) y el número de pacientes atendidos al día (OR= 1.014; IC95\%: 1.003-1.025)

\begin{tabular}{|c|c|c|c|}
\hline Variable independiente & p & Exp $\beta$ & IC95\% Ехрß \\
\hline Años trabajados en A. Primaria & 0.116 & 1.035 & $0.992-1.080$ \\
\hline Edad & 0.618 & 0.989 & $0.947-1.033$ \\
\hline Centro de Salud Docente (S/N) & 0.057 & 0.593 & $0.343-1.016$ \\
\hline Categoría profesional & 0.013 & 3.833 & $1.334-11.012$ \\
\hline Tipo de contrato & 0.626 & 1.016 & $0.145-7.116$ \\
\hline$N^{\circ}$ pacientes/dia & 0.014 & 1.014 & $1.003-1.025$ \\
\hline
\end{tabular}

Tabla VI. Modelo de regresión logística. Variable dependiente: SMG agrupada

(Satisfechos/nsatisfechos).Pruebade Hosmery Lemeshow ( $\rho=0.881$ )

Si procedemos a analizar las diferentes dimensiones que componen el cuestionario de Font Roja AP en relación con el resto de variables independientes, nos encontramos las siguientes relaciones estadísticamente significativas:

- La dimensión "Monotonía laboral" se relaciona con la categoría profesional ( $p=$ 0.026 ) $48,15 \%$ de los administrativos puntúan alto en la misma (consideran el trabajo muy monótono), frente al $27 \%$ de los médicos de familia.

- La dimensión "Estatus profesional" se relaciona con la categoría profesional $(p<0.001)$ estando insatisfechos con la misma el $75 \%$ de los administrativos frente al $14,7 \%$ de las enfermeras; esta dimensión también se relaciona con tener o no hijos $(p=0.016)$, estando más insatisfechos $(37.7 \%)$ los que tienen hijos frente a los que no (27.4\%).

- La dimensión "Relación interpersonal con compañeros" se produce una asociación con la categoría profesional $(p=0.03)$ y con el tipo de contrato $(p=$ 0.036). Así están insatisfechos el $7,7 \%$ de los administrativos y el $1.8 \%$ de los médicos de familia, y el $5.2 \%$ de los que tienen un contrato estable y $0 \%$ de los que son eventuales.

- La dimensión "Relación interpersonal con los jefes" observamos una asociación con la categoría profesional ( $p=0.007$ ), estando insatisfechos un $29.5 \%$ de los médicos frente al $12.7 \%$ de las enfermeras.

- La "Promoción profesional" se relaciona con la categoría profesional $(p<0.001)$, el tipo de contrato $(p<0.001)$ y el sexo $(p=0.03)$. Así se observa que están insatisfechos el $80.8 \%$ de los administrativos frente al $43.1 \%$ de las enfermeras, el $56.3 \%$ de los que tienen contrato estable, $54.8 \%$ de los eventuales, $11.1 \%$ de los MIR/EIR, y el $49.4 \%$ de los hombres frente al $55.6 \%$ de las mujeres.

- La "Presión en el trabajo" se relaciona con la categoría profesional $(p<0.001)$ estando insatisfechos el $31.3 \%$ de los médicos frente al $14.7 \%$ de las enfermeras o el $7.7 \%$ de los administrativos.

- La "Competencia profesional" se relaciona con el tipo de contrato $(p=0.026)$ estando insatisfechos el $32.3 \%$ de los eventuales frente al $17.8 \%$ de los estables.

- La "Tensión relacionada con el trabajo" se relaciona con la categoría profesional $(p<0.001)$, estando insatisfechos el $85.7 \%$ de los médicos frente al $53.8 \%$ de los administrativos.

- La "Satisfacción por el trabajo" se relaciona con la categoría profesional $(p<0.001)$ y con el tipo de contrato $(p=0.041)$; estando más satisfechos el $95.1 \%$ de las enfermeras, $100 \%$ de los MIR/EIR frente al $61.5 \%$ de los 
administrativos, y el $86.3 \%$ de los propietarios e interinos frente al $74.2 \%$ de los eventuales.

\section{Análisis del comportamiento de las variables en el personal de enfermería.}

La edad media de los profesionales de enfermería fue 52,6 años (Desviación típica: 11.1). El $80,6 \%$ son mujeres, $61,2 \%$ casados o con pareja estable, el $64,1 \%$ tienen hijos. El 74,8\% son estatutarios fijos, y llevan una media de 15,3 años trabajando en atención primaria. El $52.4 \%$ trabajan en centros de salud no docentes. La presión asistencial media que tienen es de 21,85 pacientes al día. 91,3\% de las enfermeras se autodeclaran en general satisfechas con el trabajo; y según el Cuestionario de Font-Roja el $70.9 \%$ están satisfechos.

Las dimensiones de mayor satisfacción son: "Relación interpersonal con los compañeros" (79.6\%), "relación interpersonal con los jefes" (73.8\%), "competencia profesional" (71.8\%) y estatus profesional $(61,2 \%)$. Las dimensiones que mayor insatisfacción generan son: "promoción profesional" (42.7\%), "presión en el trabajo" (35\%) y "monotonía laboral" (29.1\%)

Si realizamos un análisis univariante por cada una de las dimensiones, encontramos que la dimensión monotonía en el trabajo genera mayor insatisfacción a mayor edad (56.5 vs 49.32, $\mathrm{p}=0.02$ ). La dimensión promoción profesional genera mayor insatisfacción a mayor edad (56 vs 47.95, $\mathrm{p}=0.004$ ), a más años trabajados en atención primaria aunque no llega a ser estadísticamente significativa (17.7 vs 12.54 , $\mathrm{p}=0.07)$, y a disponer de un contrato estable frente a eventual $(46.7 \%$ vs $10 \%, p=$ 0.048). La dimensión presión en el trabajo genera mayor insatisfacción a mayor número de pacientes atendidos al día (24.34 vs 19.92, $\mathrm{p}=0.001)$. La dimensión tensión en el trabajo genera más insatisfacción a mayor edad (54.49 vs $52.03, \mathrm{p}=$ $0.006)$, a mayor número de pacientes $(22.34$ vs $19.58, \mathrm{p}=0.027)$, y a disponer de un contrato estable $(62.2 \%$ vs $40 \%, p=0.028)$. La dimensión estatus profesional genera mayor satisfacción a menor edad $(50.019$ vs $57.57, \mathrm{p}=0.024)$. En el resto de dimensiones (relación interpersonal con compañeros y jefes, competencia profesional) no se observan diferencias estadísticamente significativas.

\section{DISCUSIÓN}

La tasa de participación es similar a otros estudios realizados en nuestra comunidad autónoma ${ }^{18,19}$ los cuales lograron tasas de respuesta del 61,35\% y 54\% respectivamente; similar al $57.7 \%$ alcanzado por nuestro estudio. Esta tasa de participación es algo inferior a estudios realizados en otros lugares de nuestro país $14,16,17,20,21$. A pesar de ello entendemos que es suficiente para que los resultados sean representativos de la población estudiada.

Resulta prácticamente imposible poder hacer una comparación entre los resultados de los diferentes estudios al existir una gran variabilidad en la forma en que se había analizado el cuestionario de Font-Roja AP.

El bajo grado de acuerdo existente entre el resultado del cuestionario (SMG) y la satisfacción autodeclarada por el profesional en pregunta cerrada nos orienta a pensar que, quizás, siendo la satisfacción laboral tan subjetiva y personal, las preguntas cerradas con solo dos opciones no son adecuadas como instrumento de 
valoración y deberíamos utilizar cuestionarios que investiguen en un mayor número de dimensiones o matices y con una cierta gradación de posibilidades (escalas Likert). De los resultados obtenidos podemos dibujar un perfil del profesional insatisfecho: médico varón, de mayor edad, separado y con hijos, que tiene un contrato eventual en un centro de salud no docente y que lleva más años trabajando en atención primaria y con alta presión asistencial en la consulta. Por el contrario el perfil del profesional satisfecho sería el de una mujer, enfermera, joven, soltera y sin hijos, con plaza en propiedad que trabaja en un centro de salud docente, que lleva pocos años trabajando en atención primaria y con baja presión asistencial en la consulta.

Estos perfiles coinciden en gran medida con los expuestos en trabajos publicados anteriormente, así en nuestra comunidad se publicó un trabajo ${ }^{19}$ donde la edad era una variable relevante en la satisfacción autodeclarada, siendo los jóvenes los que mostraban mayor sensación de posibilidad de mejora, expectativas y posibilidades de aprender cosas nuevas.

Aún así de todas las variables hemos podido observar que las que tienen un mayor peso son el número de pacientes atendidos al día, y la categoría profesional; generando una mayor insatisfacción a mayor número de pacientes; y la categoría profesional siendo 3,8 veces más probable estar insatisfecho si eres médico.

En relación con las dimensiones valoradas en el cuestionario Font-Roja podemos observar que las dimensiones peor valoradas son "Tensión en el trabajo", "Promoción profesional" y "Presión del trabajo", dimensiones que en gran medida coinciden con lo encontrado en estudios previos ${ }^{5,16,17,18,22}$. Es conocido que la tensión laboral puede ser fuente de estrés en el individuo, y puede estar provocada por múltiples factores como son la carga de trabajo, el hacer frente a las expectativas del paciente, el estado de enfermedad-muerte ligado a estas profesiones, etc. En nuestro caso, uno de los motivos de esta mala valoración por parte de los facultativos podría ser la presión asistencial y la responsabilidad en la toma de decisiones clínicas, entre otras. En relación con la "Promoción profesional" tenemos que tener en cuenta que nuestro estudio se realiza en centros sanitarios públicos, y los profesionales son estatutarios; teniendo en cuenta esto, la promoción profesional ha sido una preocupación para la administración sanitaria desde hace tiempo, siendo la carrera y desarrollo profesional su mayor apuesta por mejorar esta dimensión en personal estatutario fijo (que son los que alcanzan peor puntuación en esta dimensión), pero como vemos parece que ha resultado infructuosa. En relación con la promoción profesional, nos llama la atención que se produce una peor valoración (con diferencias significativas) en las mujeres que en los hombres, y esto en un sector con amplia mayoría de mujeres. Entendemos que esta diferencia en la percepción merecería de mayor número de estudios para valorar si se están produciendo políticas de promoción que marginen el desarrollo futuro de las mujeres en los centros sanitarios públicos.

Las dimensiones que han alcanzado un puntuación más alta en el grado de satisfacción han sido la "satisfacción por el trabajo" y la "relación interpersonal con los compañeros". Esta última dimensión concuerda con lo visto en el trabajo realizado en Asturias en el año $2013^{18}$. Los investigadores de dicho trabajo utilizaron otro cuestionario de valoración diferente al nuestro, pero que incluye un ítem que se podría asemejar que es "Conflictos con otras personas de mi trabajo", al que los participantes otorgaron una baja puntuación (3.23 sobre 10). Este dato es importante porque se conoce que una buena relación entre compañeros es un factor de protección frente al estrés laboral, aumenta el grado de satisfacción y la ilusión por el trabajo. En 
contrapartida, una mala relación en los equipos de Atención Primaria también se ha descrito como un factor de riesgo para el desgaste ${ }^{21}$. En relación con la dimensión "satisfacción en el trabajo" si observamos los ítems que la componen los dos que obtienen mayor puntuación son "Tengo interés por las cosas que realizo" y " tengo la sensación que lo que hago vale la pena" podríamos pensar que el trabajo que se desarrolla en los centros de salud tiene un componente motivador per se para los profesionales; y quizás se confirman como elementos clave a reforzar para incrementar la satisfacción, la relevancia y significación del trabajo para los ciudadanos.

En relación con la valoración que los profesionales realizan de su capacitación nos encontramos que se obtienen puntuaciones positivas. Así el ítem "Con frecuencia siento no estar capacitado para mi trabajo" tiene una puntuación media de 1.97 (siendo 1 totalmente en desacuerdo con la afirmación); este dato coincide con investigaciones previas ${ }^{5,17,18,22}$. Todos los colectivos reflejan sentirse competentes en sus labores, lo que pudiera tener relación con el hecho de que se trata de colectivos, en general, muy cualificados, donde predominan las personas con niveles educativos superiores y, en muchos casos, formación especializada. Este sentimiento de alta capacitación puede estar relacionado también con que la mayoría de nuestros trabajadores son fijos con una amplia experiencia en el campo de la Atención Primaria; o también con un sesgo de autovaloración.

Para finalizar, nos parece de interés reflejar que al cruzar las diferentes dimensiones con el resto de variables independientes, observamos que cada dimensión se relaciona con unas variables diferentes y con unas categorías diferentes. Esto nos puede dar una pista sobre los matices de la insatisfacción según el perfil profesional. Por ejemplo, observamos que la monotonía se relaciona con la categoría profesional, pero puntúan bien los médicos y muy mal los administrativos, y sin embargo, en la presión en el trabajo es al contrario. Lo que nos lleva a pensar que las estrategias para incrementar la satisfacción del profesional deben contemplar perfiles diferentes.

Parece imprescindible y urgente para nuestra organización comenzar a diseñar estrategias que, en primer lugar, valoren la satisfacción del profesional y las variables asociadas y después, establezcan acciones de mejora que deberían ser dinámicas, continuas en el tiempo y múltiples para ajustarse a los diferentes profesionales existentes y a los diferentes momentos vitales. Las políticas genéricas para todos los profesionales no servirán para mejorar la satisfacción global en la organización muy probablemente.

Observando las dimensiones más afectadas, parece que las estrategias deberían ir orientadas a mejorar la promoción profesional, reducir la monotonía laboral y la presión y tensión en el trabajo, quizás diseñar puestos laborales que a partir de unos años de experiencia comiencen a combinar tareas asistenciales con otro tipo de tareas (investigación, docencia, gestión y planificación, intervención comunitaria) reduciendo el tiempo dedicado a la asistencia y sustituyéndolo por tareas en relación con esas áreas podría ayudar a incrementar la satisfacción laboral de las enfermeras. También reflexionar sobre la posibilidad de introducir días de permiso vinculados a la presión en el trabajo, y no solo a la antigüedad como ocurre ahora.

\section{CONCLUSIONES}

- La satisfacción laboral es una dimensión personal que requiere el abordaje mediante cuestionarios que valoren de forma gradual diferentes dimensiones. 
- La mayoría de los profesionales se declaran satisfechos; aún así, hay un alto porcentaje de insatisfacción y constituye un problema relevante para la organización.

- Sería necesario que la administración analizase, de forma rutinaria, la satisfacción de los profesionales, valorando tanto aspectos que generan insatisfacción como satisfacción (como la relevancia del trabajo) por categoría profesional y contemplando otras variables de índole social.

- En la promoción profesional debemos prestar atención a la variable de género, realizando estudios específicos en primer lugar y estableciendo mecanismos correctores si fuera preciso.

- Entendemos que la satisfacción laboral es una cuestión dinámica que va cambiando según cambian otros factores, debemos establecer mecanismos de valoración de forma continua a lo largo de la vida profesional.

- Del citado análisis se deberían derivar estrategias para incrementar la satisfacción; sino personalizadas, al menos si por perfiles.

- Los aspectos generales que más influyen en la insatisfacción son la categoría profesional y la presión asistencial.

- Las categorías que mayor satisfacción apuntan son los profesionales en formación y el personal de enfermería. Las más insatisfechas, médicos y personal administrativo, aunque por motivos diferentes.

- Hay que buscar estrategias que permitan el desarrollo profesional variando las funciones y tareas, reduciendo la carga asistencial e introduciendo otras áreas de interés como investigación, gestión y planificación, docencia e intervención comunitaria para los profesionales.

- Otras posibilidades pasarían por el diseño de jornadas laborales en el que se permitan más días de permisos asociados a presión en el trabajo, y no solo a antigüedad.

\section{REFERENCIAS}

1. Fernández MI, Villagrasa JR, Gamo MF, Vázquez J, Cruz E, Aguirre MV, et al. Estudio de la satisfacción laboral y sus determinantes en los trabajadores sanitarios de un área de Madrid. Rev Esp Salud Pública 1995, Vol 69, N6: 487-497.

2. Mayo E. The social problems of an industrial civilization. Cambridge, Mass: Harvard University Press, 1945

3. Maslow AH. Motivation and personality. $2^{\text {nd }}$ ed. Nueva York: Harper and Bros. Publishers, 1970.

4. Herzberg F. The motivation to work. $2^{\text {nd }}$ ed. Nueva York: John Wiley and Sons, 1959.

5. López-Soriano F, Bernal L, Cánovas A. Satisfacción laboral de los profesionales en un Hospital Comarcal de Murcia. Rev Calidad Asistencial. 2001;16:243-246.

6. Evolución de la enfermería hacia la satisfacción profesional [editorial].Rev Esp Sanid Penit 2009; 11:65-67.

7. Ramírez-Sánchez TS, Nájera-Aguilar P, Nigenda-López G. Percepción de la calidad de la atención de los servicios de salud en México: perspectiva de los usuarios. Salud Pública de Méx 1998;40(1):3-12.

8. Hernández LB, García PC, et al. Satisfacción de usuarios en unidades de medicina familiar. Rev Med IMSS 2002. 40(5):373-378.

9. Ponce-Gómez J, Reyes-Morales H, Ponce-Gómez G. Satisfacción laboral y calidad de atención en enfermería en una unidad médica de alta especialidad. Rev Enferm IMSS 2006;14(2):65-73. 
10. Parada ME, Moreno R, Mejías Z, Rivas A, Rivas F, Cerrada J, et cols. Satisfacción laboral y síndrome de burnout en el personal de enfermería del Instituto Autónomo Hospital Universitario Los Andes, Mérida, Venezuela. Revista Facultad Nacional de Salud Pública 2005. 23(1):33-45.

11. Ibern P. La medida de la satisfacción en los servicios sanitarios. Gac Sanit 1992;6:176-85.

12. Hyrkas K, Appelqvist-Schmidlechner K, Haataja R. Efficacy of clinical supervision: influence on job satisfaction, burnout and quality of care. J Adv Nurs 2006; Aug; 55(4): 521-35.

13. Fernández G. Satisfacción laboral y salud psíquica en el mundo sanitario, ¿un lujo?. Todo Hospital 1995;118:29-36.

14. Vera-Remartínez EJ, Mora LM, González JA, García J, Garcés E, Domínguez JA, et al. Satisfacción laboral de la enfermería en las prisiones españolas. Rev Esp Sanid Penit 2009; 11:80-86.

15. Peya M. Satisfacción laboral: una breve revisión bibliográfica. Nursing 2008; 26(2): 62-65.

16. Fernández MI, Villagrassa JR, Gamo MF, Vázquez J, Cruz E, Aguirre MV et cols. Estudio de la satisfacción laboral y sus determinantes en los trabajadores sanitarios de un Área de Madrid. Rev Esp Salud Pública 1995; 69(6): 487-497.

17. Fernández MI, Moinelo A, Villanueva A, Andrade C, Rivera M, Gómez JM et cols. Satisfacción laboral de los profesionales de Atención Primaria del Área 10 del Insalud de Madrid. Rev Esp Salud Pública 2000; 74(2): 139-147.

18. Díaz C, Suárez O, Fueyo A, Mola P, Rancaño I, Sánchez AM, et cols. Calidad de vida de los profesionales en el modelo de Gestión Clínica de Asturias. Gac Sanit 2013; 27(6): 502-507.

19. Olivar C, González S, Martínez MM. Factores relacionados con la satisfacción laboral y el desgaste profesional en los médicos de Atención Primaria de Asturias. Aten Primaria 1999: 24: 352-9.

20. Lomeña JA, Campaña FM, Nuevo G, Rosas D, Berrocal A, García F. Burnout y satisfacción laboral en Atención primaria. Medicina de Familia 2004; 5(3): 147-155.

21. Sobrequés J, Cebriá J, Segura J, Rodríguez C, García M, Juncosa S. La satisfacción laboral y el desgaste profesional de los médicos de atención primaria. Aten Primaria 2003; 31(4):227-33.

22. Pérez MT, Díaz C. Satisfacción laboral en enfermeros/as de Atención Primaria del Servicio Gallego de Salud. Metasdeenfermería 2007; 10(10):6-10.

23. Fernández AM, Santa Clotilde E, Casado MI. Calidad de vida profesional de los profesionales de enfermería en Atención Primaria de Soria. Biblioteca Lascasas 2007; 3(1). Disponible en http://www.indexf.com/lascasas/documentos/lc0213.php

24. Aranaz J, Mira J. Cuestionario Font Roja. Un instrumento de medida de la satisfacción en el medio hospitalario. Todo Hosp. 1988; 52: 63-66.

ISSN 1695-6141

(c) COPYRIGHT Servicio de Publicaciones - Universidad de Murcia 